\title{
Evaluación in vitro de la actividad anti Trypanosoma cruzi de aceites esenciales de diez plantas medicinales
}

\author{
In vitro anti-Trypanosoma cruzi activity of essential oils of ten medicinal plants
}

\author{
Juan Rojas ${ }^{1}$, Hilda Solís ${ }^{2}$, Olga Palacios ${ }^{2}$ \\ ${ }^{1}$ Instituto de Investigaciones Clínicas, Facultad de Medicina, UNMSM. Lima, Perú. \\ ${ }^{2}$ Instituto de Medicina Tropical, Facultad de Medicina, UNMSM. Lima, Perú.
}

\begin{abstract}
Resumen
Objetivos: Determinar la actividad anti Trypanosoma cruzi in vitro de los aceites esenciales de 10 plantas medicinales. Además, determinar la actividad citotóxica de los aceites contra células de mamíferos y la actividad modulatoria de los aceites sobre el óxido nítrico. Diseño: Estudio experimental in vitro. Institución: Instituto de Investigaciones Clínicas e Instituto de Medicina Tropical, Facultad de Medicina, Universidad Nacional Mayor de San Marcos, Lima, Perú. Material biológico: Epimastigotes de Trypanosoma cruzi, células Raw 264.7, aceites esenciales de Mentha X piperita L (menta), Rosmarinus officinalis L (romero), Chenopodium ambrosioides L (paico), Eucaliptus globulus Labill (eucalipto), Artemisia absinthium L (ajenjo), Melissa officinalis L (toronjil), Minthostachys setosa Brig (muña), Cimbopogon citratus (hierba luisa), Aloysia triphylla (cedrón) y Mentha spicata L (hierba buena). Método: La actividad tripanocida se evaluó contra epimastigotes cultivados en medio LIT, incubados por 48 horas a $37^{\circ} \mathrm{C}$ en incubador humidificado con $\mathrm{CO}_{2}$ al $5 \%$. El cristal violeta se utilizó como control positivo. La actividad citotóxica de los productos contra células mamíferas se evaluó en células RAW 264.7 y la actividad modulatoria de los compuestos sobre óxido nítrico también se determinó en los cultivos de células RAW 264.7. Principales medidas de resultados: Porcentaje de inhibición de viabilidad y Cl50. Resultados: Los aceites esenciales de Cymbopogon citratus (hierba luisa) y Aloysia triphylla (cedrón) inhibieron significativamente el crecimiento de la forma epimastigote de $\mathrm{T}$. cruzi, con una $\mathrm{Cl} 50$ de 63,09 y $96,49 \mu \mathrm{g} / \mathrm{mL}$, respectivamente. No hubo variación significativa de la concentración de óxido nítrico y tampoco se evidenció citotoxicidad. Conclusiones: Los aceites esenciales de Cymbopogon citratus y Aloysia triphylla mostraron actividad anti-Trypanosoma cruzi in vitro y no fueron citotóxicas para las células mamíferas.

Palabras clave: Trypanosoma cruzi; aceites esenciales; plantas medicinales.
\end{abstract}

\begin{abstract}
Objectives: To determine in vitro anti-Trypanosoma cruzi activity of essential oils of 10 plants, cytotoxic activity of oils against mammalian cells and modulatory activity of oils on nitric oxide. Design: In vitro analytical-experimental research, prospective longitudinal cutting. Setting: Institute of Clinical Research, and Institute of Tropical Medicine, Faculty of Medicine, Universidad Nacional Mayor de San Marcos, Lima, Peru. Biological material: Trypanosoma cruzi epimastigotes; Raw 264.7 cells; ten medicinal plants: Mentha X piperita L (menta), Rosmarinus officinalis $L$ (romero), Chenopodium ambrosioides $L$ (paico), Eucaliptus globulus Labill (eucalipto), Artemisia absinthium L (ajenjo), Melissa officinalis L (toronjil), Minthostachys setosa Brig (muña), Cimbopogon citratus (hierba luisa), Aloysia triphylla (cedrón), and Mentha spicata L (hierba buena). Methods: The trypanocidal activity was assessed against epimastigotes grown in LIT medium, incubated for 48 hours at $37^{\circ} \mathrm{C}$ in humidified incubator with $5 \% \mathrm{CO}_{2}$. Crystal violet was used as positive control. The cytotoxic activity of products against mammalian cells was assessed in RAW 264.7 cells and the modulatory activity of compounds on nitric oxide was also measured in cultured RAW 264.7 cells. Main outcome measures: Percentage inhibition of viability and IC50. Results: Essential oils of Cymbopogon citratus (lemon verbena) and Aloysia triphylla (lemon verbena) inhibited the growth of T. cruzi epimastigote form with good IC50s of 63.09 and $96.49 \mathrm{mg} / \mathrm{mL}$, respectively. There was no significant variation in the concentration of nitric oxide and no cytotoxicity was evident. Conclusions: Essential oils of Aloysia triphylla and Cymbopogon citratus showed anti-Trypanosoma cruzi activity in vitro and were not cytotoxic to mammalian cells.
\end{abstract}

Key words: Trypanosoma cruzi; oils, volatile; plants, medicinal.

An Fac med. 2010;71 (3):161-5

\section{INTRODUCCIÓN}

La Trypanosomiasis americana o enfermedad de Chagas es una infección parasitaria causada por Trypanosoma cruzi; es transmitida por insectos hematófagos de la familia Reduviidae, conocidos comúnmente en nuestro país como 'chirimachas' (1). Esta enfermedad afecta principalmente a la población rural y periurbana en el continente americano, donde las viviendas son construidas de adobe o carrizo y el hacinamiento favorece la presencia y proliferación del vector ${ }^{(2)}$. En el Perú, alrededor de 394000 habitantes son infestados por el vector y 24000 personas son infectadas ${ }^{(3)}$.
La enfermedad de Chagas está presente en casi todos los países de América Latina y afecta de 18 a 20 millones de personas desde México hasta Chile y Argentina. En el Perú, los departamentos en los que se ha reportado casos autóctonos de enfermedad de Chagas son: Piura, Cajamarca, Amazonas, Apurímac, San Martín, Junín, Ucayali, Huánuco, Ica, Arequipa, Moquegua y Tacna. En el país, durante el año 2006, se ha notificado 77 casos de tripanosomiasis americana o enfermedad de Chagas en el sistema nacional de vigilancia epidemiológica; los casos proceden de las regiones de Arequipa (67 casos), Cajamarca, Ica, Loreto, Moquegua y Pasco (2).
La tasa de prevalencia nacional en el Perú se estima en 0,01-0,02 x 100000 habitantes y una tasa nacional acumulada de 7,29 x 100000 . Se estima en 43170 los casos de infección por $T$. cruzi en las áreas endémicas, de las cuales 1209 corresponden a formas agudas u oligosintomáticas y 22962 a casos crónicos, siendo 3142 los niños menores de 5 años afectados en ambos grupos. El grupo de edad más afectado se encuentra entre los 20 y 54 años de edad ${ }^{(4)}$.

La farmacoterapia actual de la enfermedad de Chagas comprende escasamente dos fármacos, el nifurtimox y el benznidazol. Ambos fármacos son tóxicos; es 
necesario administrarlos por largo tiempo y solo sirven para la fase aguda de la enfermedad ${ }^{(5)}$. La falta de investigación de drogas antitripanosómicas por parte de la industria farmacéutica se refleja en el hecho que solo $1 \%$ de las nuevas drogas aprobadas entre 1975 y 1999 fueron para enfermedades tropicales ${ }^{(6)}$, por lo que la enfermedad de Chagas forma parte de una lista de infecciones conocidas como enfermedades tropicales olvidadas, con una prevalencia global de 8 a 9 millones y una población en riesgo de 25 millones de personas ${ }^{(7)}$.

Ante ello, es necesaria la búsqueda de nuevos agentes terapéuticos para la enfermedad de Chagas, siendo las plantas medicinales una alternativa viable. Así, nos propusimos realizar la presente investigación para determinar la actividad anti-Tripanosoma cruzi in vitro de los aceites esenciales de Mentha X piperita $\mathrm{L}$ (menta), Rosmarinus officinalis L (romero), Chenopodium ambrosioides L (paico), Eucaliptus globulus Labill (eucalipto), Artemisia absinthium L (ajenjo), Melissa officinalis L (toronjil), Minthostachys setosa Brig (muña), Cimbopogon citratus (hierba luisa), Aloysia triphylla (cedrón) y Mentha spicata L (hierba buena). También nos propusimos determinar el efecto sobre la producción de óxido nítrico por las células RAW 264.7, como un probable mecanismo de acción, y determinar el efecto citotóxico sobre sobre células mamíferas.

\section{MÉTODOS}

La extracción de los aceites esenciales se realizó por el método de arrastre por vapor de agua ${ }^{(8)}$, para lo cual se utilizó las hojas frescas de cada planta. El destilado se separó tomando en cuenta sus propiedades de inmiscibilidad y diferencia de densidades entre el agua y el aceite esencial, utilizando una pera de separación de vidrio. Se deshidrató las impurezas de agua en el aceite esencial con $\mathrm{Na}_{2} \mathrm{SO}_{4}$ anhidro, se filtró y se guardó en un frasco de vidrio color ámbar bajo refrigeración, a una temperatura de $4^{\circ} \mathrm{C}$, hasta su uso.

Como parásito se utilizó tripomastigotes de $T$. cruzi que fueron mantenidos en ratón mediante pasajes cada 20 días. Los epimastigotes fueron mantenidos en medio LIT (liver infusion tryptose).

Las células RAW 264.7 fueron mantenidas en medio RPMI-1640 y suplementado con suero bovino fetal al $10 \%$, a $37^{\circ} \mathrm{C}$, en un incubador humidificado con $5 \%$ de $\mathrm{CO}_{2}$.

Para la determinación de la actividad tripanocida contra epimastigotes, los aceites esenciales fueron disueltos previamente en DMSO (dimetil sulfóxido) al $1 \%$ y diluidos en medio LIT. Según el método de Araya y col. ${ }^{(9)}$, los epimastigotes mantenidos en medio LIT fueron contados en una cámara hemocitométrica de Neubauer; a continuación $50 \mu \mathrm{L}$ de una suspensión de 4 × $10^{6}$ parásitos/ $\mathrm{mL}$ fueron adicionados en microplacas de 96 pozos, luego los aceites esenciales disueltos en medio LIT, en dosis de 50, 100,250 y $500 \mu \mathrm{g} / \mathrm{mL}$, fueron adicionados en un volumen de $50 \mu \mathrm{L}$. Se utilizó cristal violeta en una concentración de $250 \mathrm{\mu g} /$ $\mathrm{mL}$ como control positivo, y medio de cultivo con DMSO al 1\% como control de viabilidad. Se incubó durante 48 horas a $28^{\circ} \mathrm{C}$. La viabilidad de los epimastigotes está basada en la conversión celular de la sal soluble de tetrazolio MTT (bromuro de 3-(4,5-dimetiltiazol-2-ilo)-2,5difeniltetrazol) en formazan insoluble por enzimas mitocondriales, para lo cual se adicionó $10 \mu \mathrm{L}$ de MTT $(5 \mathrm{mg} / \mathrm{mL})$ a cada pozo y se incubó a $37^{\circ} \mathrm{C}$ por 4 horas. La extracción del formazan se llevó a cabo con SDS (sodium dodecyl sulfate) al 10\%, por 18 horas $(100 \mu \mathrm{L} /$ pozo $)$, a $26^{\circ} \mathrm{C}$. El número de epimastigotes vivos fue determinado indirectamente por la $\mathrm{DO}$ a 570 nm, leído en un lector de microplacas Dynex Technologies Revelation $4.25^{\circledR}$. El cálculo de la CI50 (concentración requerida para 50\% de inhibición) se realizó mediante análisis de la curva sigmoide del logaritmo de la dosis versus porcentaje de viabilidad.

La viabilidad se calculó de la siguiente manera:

\section{viabilidad $(\%$ control $)=100 \times$ absorbancia de muestra/absorbancia de control}

Para la actividad citotóxica contra células mamíferas, siguiendo el método de Tempone y col. (10), $50 \mu \mathrm{L}$ de células RAW 264.7 ECACC (European collection of cell cultures), en una concentración de $4 \times 10^{5}$ células $/ \mathrm{mL}$, fueron sembradas en microplacas de 96 pozos; luego se adicionó los aceites esenciales en concentración de 100, 50 y $20 \mu \mathrm{g} / \mathrm{mL}$, disueltos en DMSO y diluidos en medio RPMI 1640 sin rojo de fenol, en un volumen de 50 $\mu \mathrm{L}$. Las microplacas fueron incubadas por 48 horas a $37^{\circ} \mathrm{C}$ en un incubador humidificado con $5 \%$ de $\mathrm{CO}_{2}$. Las células control fueron incubadas en presencia de DMSO y sin drogas. La viabilidad de las células se determinó con el ensayo MTT. Se realizó un análisis de regresión no lineal dosis-respuesta para el cálculo de CI50.

El nivel de óxido nítrico (NO) producido por macrófagos fue determinado indirectamente por la medición de la concentración de nitrito en el medio de cultivo, según el método de TorresSantos y col. ${ }^{(11)}$, con modificaciones. En una microplaca de 96 pozos se adicionó $100 \mu \mathrm{L}$ de la suspensión de 4 × $10^{6}$ células RAW 264.7 y se incubó a $37^{\circ} \mathrm{C}$, por 2 horas, en $5 \%$ de $\mathrm{CO}_{2}$. Después de lavar las células no adherentes, se adicionó $200 \mu \mathrm{L}$ de medio conteniendo concentraciones variadas de las sustancias de prueba, en triplicado. Después de 48 horas de incubación a $37^{\circ} \mathrm{C}$, se colectó $100 \mu \mathrm{L}$ del sobrenadante del cultivo. El contenido de nitrito se determinó con el reactivo de Griess [ácido sulfanílico al $1 \%$ en ácido fosfórico al 5\% (reactivo A) y $\mathrm{N}-1$-naftilenodiamina al 0,1\% en agua (reactivo B)], que conduce a la formación de cromóforos magenta caracterizado por color grisáceo, según el método de Miranda y col. ${ }^{(12)} \mathrm{A}$ los $100 \mu \mathrm{L}$ de sobrenadante se adicionó $50 \mu \mathrm{L}$ de reactivo A y $50 \mu \mathrm{L}$ de reactivo $B$ y después de 30 minutos se procedió a la lectura de la absorbancia en un lector de microplacas Dynex Technologies Revelation $4.25^{\circledR}$, a una longitud de onda de $540 \mathrm{~nm}$. Para el cálculo de la concentración de nitrito se utilizó una curva de calibración estándar.

Los datos obtenidos fueron expresados como media \pm desviación estándar y las comparaciones entre los grupos experimentales fueron realizadas por ANOVA de una vía seguido, por una 
prueba post-hoc de Scheffé. Las medias fueron consideradas significativamente diferentes cuando $p<0,05$. Se utilizó el software SPSS 15.0.

\section{RESULTADOS}

Los aceites esenciales de Cimbopogon citratus (hierba luisa) y Aloysia triphylla (cedrón) inhibieron el crecimiento de la forma epimastigote de $T$. cruzi con una buena CI50 (concentración requerida para 50\% de inhibición) de 63,09 y 96,49 $\mu \mathrm{g} / \mathrm{mL}$, respectivamente $(p<0,05)$. Mentha spicata (hierba buena), Chenopodium ambrosioides (paico), Rosmarinus officinalis (romero) y Eucaliptus globulus (eucalipto) exhibieron moderada actividad inhibitoria, con CI50 de 120,23 a 346,74 $\mu \mathrm{g} / \mathrm{mL}$. Por su parte, Melissa officinalis (toronjil), Mentha X piperita (menta), Minthostachys setosa (muña) y Artemisia absinthium (ajenjo) fueron inactivas (tabla 1).

Todos los aceites esenciales ensayados demostraron falta de toxicidad para las células mamíferas, toda vez que su CI50 fue $>100 \mu \mathrm{g} / \mathrm{mL}$.

El nivel de nitrito en el sobrenadante de los cultivos de células RAW 264.7 no mostró diferencia significativa por efecto de ninguno de los diez aceites esenciales, con respecto al control (figura 1); sin embargo, con Cimbopogon citratus y Aloysia triphylla se observó una disminución desde 5,10 $\pm 0,56 \mu \mathrm{M}$ (control) hasta $3,40 \pm 1,25 \mu \mathrm{M}$ y $3,00 \pm 0,82 \mu \mathrm{M}$, respectivamente.

\section{DISCUSIÓN}

En la búsqueda de nuevas drogas alternativas para el tratamiento de la enfermedad de Chagas, los productos vegetales han demostrado poseer importante actividad contra parásitos hemoflagelados. Se ha reportado que el extracto de la hoja de B. dracunculifolia, a una concentración de $3 \mathrm{mg} / \mathrm{mL}$, produjo $100 \%$ de lisis de formas tripomastigotes de T. cruzi ${ }^{(13)} ;$ los extractos y fracciones de A. amazonicus mostraron lisis de las formas tripomasigotes de $T$. cruzi in vitro ${ }^{(14)}$; otros investigadores también han demostrado el efecto tripanocida de diversos extractos vegetales (10,15,16), así como de diversas estructuras terpénicas contra uno o todos los estadíos de T. cruzi ${ }^{(17-20)}$. También han mostrado efecto tripanocida algunos alcaloides ${ }^{(21-23)}$, flavonoides ${ }^{(24-26)}$, lignanos ${ }^{(27)} \mathrm{y}$ artemisininas ${ }^{(28)}$.

En la presente investigación, los aceites esenciales de Cimbopogon citratus (hierba luisa) y Aloysia triphylla (cedrón) inhibieron el crecimiento de la forma epimastigote de Trypanosoma cruzi, con una buena CI50 $<100 \mu \mathrm{g} / \mathrm{mL}$ (tabla 1). Los aceites esenciales de otras plantas también han demostrado poseer actividad contra $T$. cruzi; así, el aceite esencial de Origanum vulgare (orégano) inhibió el crecimiento de epimastigotes de T. cruzi y también indujo lisis de tripomastigotes; semejante actividad se observó con el aceite esencial de Thymus vulgaris L (tomillo) (29). Además, los aceites esenciales de Achillea millefolium L (milenrama), Syzygium aromaticum L (clavo) y Ocimun basilicum L (albahaca) también han demostrado su actividad inhibitoria del desarrollo de epimastigotes y tripomastigotes de $T$. cruzi ${ }^{(30)}$. Es probable que la actividad tripanocida de los aceites esenciales de Cimbopogon citratus y Aloysia triphylla se encuentre relacionada con su componente principal: el citral (una mezcla de geranial y neral), dado que esta es una reconocida sustancia con propiedades antibacterianas y antiparasitarias (antimalárica) (31).

Se ha descrito varios mecanismos por los cuales los productos derivados de las plantas pueden ejercer su efecto anti-Trypanosoma cruzi. Los alcaloides isoquinolínicos están implicados fuertemente en la inhibición de una enzima antioxidante esencial del Trypanosoma, la tripanotiona reductasa. Esta enzima por sí misma ejecuta una variedad de funciones y protege al parásito de varias especies reactivas de oxígeno generados por las

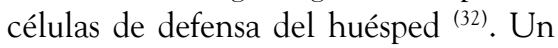
naftoimidazol derivado de $\beta$-lapachona produjo condensación anormal de cromatina y daño mitocondrial; así, los epimastigotes presentaron alteraciones de reservosomas y los tripomastigotes, una alteración en la densidad electrónica de ácidocalcisomas ${ }^{(33)}$. La artemisinina inhibe la actividad de la ATPasa dependiente de calcio en membranas de T. cruzi, sugiriendo un modo de acción

Tabla 1. Efecto de los aceites esenciales sobre epimastigotes de Trypanosoma cruzi y su toxicidad contra células mamíferas.

\begin{tabular}{|c|c|c|c|c|c|c|}
\hline \multirow[t]{2}{*}{ Aceite esencial } & \multicolumn{4}{|c|}{$\%$ de viabilidad de epimastigotes \pm DE } & \multirow{2}{*}{$\begin{array}{c}\text { Cl50 } \\
(\mu \mathrm{g} / \mathrm{mL})\end{array}$} & \multirow{2}{*}{$\begin{array}{l}\text { Citotoxicidad en células } \\
\text { RAW } 264,7(\mathrm{Cl} 50 \mu \mathrm{g} / \mathrm{mL})\end{array}$} \\
\hline & $50 \mu \mathrm{g} / \mathrm{mL}$ & $100 \mu \mathrm{g} / \mathrm{mL}$ & $250 \mu \mathrm{g} / \mathrm{mL}$ & $500 \mu \mathrm{g} / \mathrm{mL}$ & & \\
\hline Mentha spicata & $88,61 \pm 3,70$ & $51,90 \pm 1,87$ & $45,57 \pm 1,93$ & $35,44 \pm 2,40$ & 120,23 & 512,86 \\
\hline Melissa officinalis & $100,00 \pm 0$ & $100,00 \pm 0$ & $82,28 \pm 4,33$ & $73,42 \pm 4,27$ & 2511,89 & $>3000$ \\
\hline Cimbopogon citratus & $74,68 \pm 4,47$ & $29,11 \pm 0,99$ & $13,92 \pm 1,55$ & $0,00 \pm 0$ & 63,09 & 354,81 \\
\hline Chenopodium ambrosioides & $100,00 \pm 0$ & $79,75 \pm 1,84$ & $50,63 \pm 5,28$ & $37,97 \pm 4,20$ & 263,03 & 245,47 \\
\hline Mentha X piperita & $100,00 \pm 0$ & $98,73 \pm 1,78$ & $86,08 \pm 1,76$ & $78,48 \pm 2,79$ & 6918,31 & 251,19 \\
\hline Minthostachys setosa & $100,00 \pm 0$ & $100,00 \pm 0$ & $94,94 \pm 4,84$ & $91,14 \pm 2,55$ & $>100000$ & 165,96 \\
\hline Rosmarinus officinalis & $100,00 \pm 0$ & $96,20 \pm 3,62$ & $55,7 \pm 2,98$ & $44,30 \pm 3,80$ & 346,74 & 186,21 \\
\hline Eucaliptus globulus & $100,00 \pm 0$ & $60,76 \pm 2,16$ & $37,97 \pm 2,49$ & $25,32 \pm 2,43$ & 151,36 & 218,78 \\
\hline Aloysia triphylla & $89,87 \pm 2,85$ & $49,37 \pm 4,43$ & $21,52 \pm 2,59$ & $0,00 \pm 0$ & 95,49 & 257,04 \\
\hline Artemisia absinthium & $100,00 \pm 0$ & $94,94 \pm 1,86$ & $87,34 \pm 2,8$ & $83,54 \pm 3,10$ & $>100000$ & 1862,09 \\
\hline
\end{tabular}

El control positivo, cristal violeta a $250 \mu \mathrm{g} / \mathrm{mL}$, mostró $0 \%$ de viabilidad. El control negativo, medio LIT más DMSO al 1\%, mostró 100\% de viabilidad. La actividad tripanocida fue expresada como porcentaje de viabilidad de las formas de epimastigote de $T$. cruzi \pm desviación estándar. 


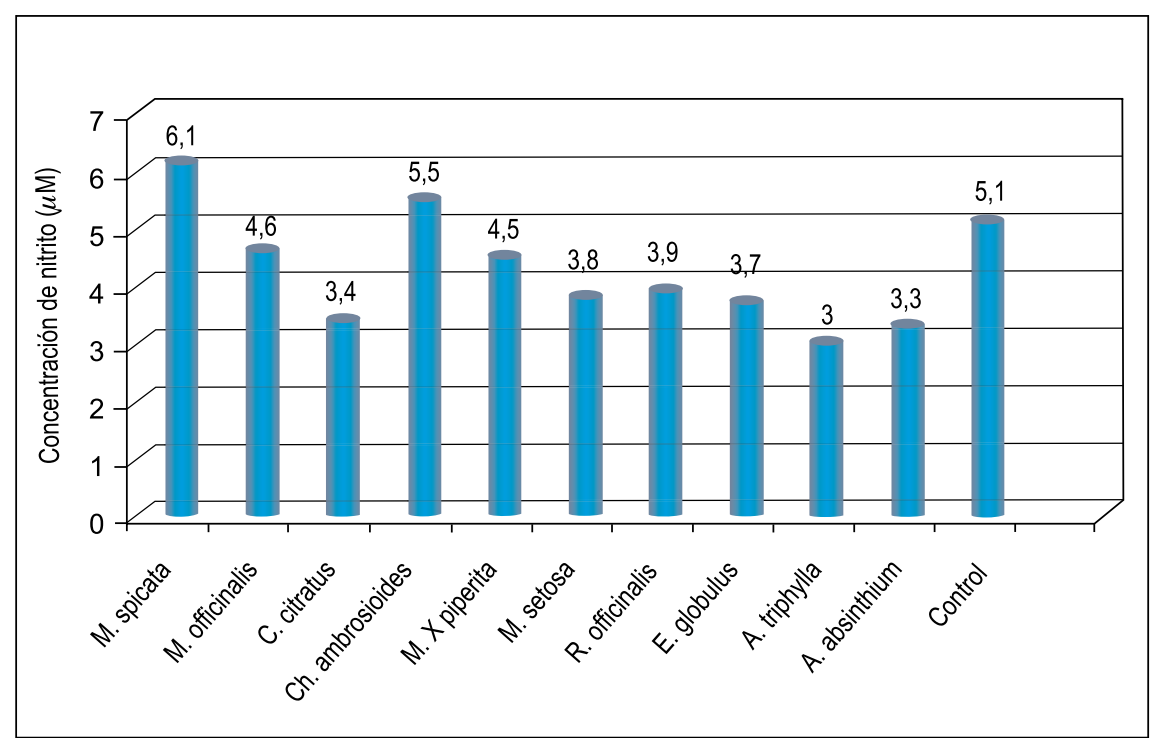

Figura 1. Efecto de $125 \mu \mathrm{g} / \mathrm{mL}$ de aceites esenciales sobre la producción de nitrito $(\mu \mathrm{M})$ en células RAW 264.7.

vía bombas de membrana ${ }^{(28)}$. Además, el diterpeno komaroviquinona mostró potente actividad tripanocida, debido a la generación de especies reactivas de oxígeno catalizada por enzimas de $T$. cruzi ${ }^{(34)}$.

Existen evidencias experimentales que demuestran que el óxido nítrico está involucrado en la actividad microbicida de macrófagos contra varios patógenos intracelulares, incluyendo Leishmania major, Trypanosoma cruzi, Toxoplasma gondii; así como también que los eosinófilos tienen actividad microbicida contra muchos parásitos, tales como Schistosoma mansoni, Trichinella spiralis, T. cruzi y $L$. amazonensis ${ }^{(35)}$. En un intento por determinar el mecanismo de acción tripanocida, nosotros evaluamos la producción de óxido nítrico por las células RAW 264.7 por efecto de los aceites esenciales; sin embargo, no encontramos diferencia significativa con respecto al control. Por el contrario, en el caso de los aceites de hierba luisa y cedrón encontramos una disminución del nivel de óxido nítrico (figura 1), lo cual guarda relación con los hallazgos de Jeon $\mathrm{H}$ y col. ${ }^{(36)}$, quienes demostraron que el citral, principal componente de ambos aceites esenciales, produjo inhibición de la producción de óxido nítrico. Esto nos indicaría que probablemente se encuentre implicado otro mecanismo de acción, diferente al óxido nítrico, tal como lo demuestra un estudio mediante microscopia electrónica, donde se observó que los amastigotes de Trypanosoma cruzi fueron desintegrados por los macrófagos peritoneales de ratón y que el peróxido de hidrógeno fue la molécula efectora responsable de la acción tripanocida de los macrófagos ${ }^{(37)}$.

De igual manera, los aceites esenciales ensayados no han demostrado tener efecto citotóxico sobre células mamíferas in vitro, lo cual nos podría sugerir falta de toxicidad para futuros estudios preclínicos in vivo.

Concluimos de la presente investigación que, en condiciones experimentales, los aceites esenciales de Cimbopogon citratus (hierba luisa) y Aloysia triphylla (cedrón) mostraron efecto anti- Trypanosoma cruzi in vitro y no fueron citotóxicas para las células mamíferas.

Trabajo de investigación financiado por el Consejo Superior de Investigaciones de la UNMSM.

\section{REFERENCIAS BIBLIOGRÁFICAS}

1. Ministerio de Salud. Enfermedad de Chagas. Oficina General de Epidemiología, Instituto Nacional de Salud. Lima. 2001.
2. Ministerio de Salud. Dirección General de epidemiología. Boletín epidemiológico (Lima), 2006;15 (33):5.

3. Guhl F. Chagas disease in Andean countries. Mem Inst Oswaldo Cruz. 2007;102 Suppl I:29-37.

4. Ministerio de Salud. Doctrina, Normas y Procedimientos para el Control de la Tripanosomiasis o Enfermedad de Chagas en el Perú. Dirección General de Salud de las Personas. Programa de Control de Malaria y otras enfermedades Metaxénicas. Lima. Perú. 1998.

5. Brunton L, Lazo J, Parker K. Goodman \& Gilman Las Bases Farmacológicas de la Terapéutica. $11^{\text {a }}$ ed. Colombia: McGraw-Hill Interamericana. 2007:1049-62.

6. Ford N, Torreele E. Neglected diseases of global importance. JAMA. 2001;286(23):2943-4.

7. Hotez P, Molyneux D, Fenwick A, Kumaresan J, Ehrlich S, Sachs J, et al. Control of neglected tropical diseases. N Engl J Med. 2007;357:1018-27.

8. Lahlou M. Methods to study the phytochemistry and bioactivity of essential oils. J Phytother Res. 2004;18:435-48.

9. Araya J, Neira I, Da Silva S, Mortara R, Manque $\mathrm{P}$, Cordero E, et al. Diterpenoids from Azorella compacta (Umbelliferae) active on Trypanosoma cruzi. Mem Inst Oswaldo Cruz, Rio de Janeiro. 2003;98(3):413-8.

10. Tempone A, Treiger Borborema S, De Andrade H, De Amorim Gualda N, Salerno Carvalho C, Bachiega D, et al. Antiprotozoal activity of Brazilian plant extracts from isoquinoline alkaloid-producing familias. Phytomedicine. 2005;12:382-90.

11. Torres-Santos E, Lopes D, Rodrigues Oliveira R, Carauta J, Bandeira Falcao C, Kaplan M, Rossi-Bergmann. Antileishmanial activity of isolated triterpenoids from Pourouma guianensis. Phytomedidicne. 2004;11:114-20.

12. Miranda K, Espey M, Wink D. A rapid, simple spectrophotometric method for simultaneous detection of nitrate and nitrite. Nitric Oxide. 2001;5(1):62-71.

13. Da Silva A, Pires P, Gregório L, Silva A, Albuquerque $S$, Bastos J. In vitro trypanocidal activity evaluation of crude extract and isolated compounds from Baccharis dracunculifolia D. C. (Asteraceae). I Pharm Pharmacol. 2004;56:1195-9.

14. Rosas L,Campos F, Nascimento S, Januário $A$, FranÇa $S$, Nomizo A, et al. In vitro evaluation of the cytotoxic and trypanocidal activities of Ampelozizyphus amazonicus (Rhamnaceae). Braz J Med Biol Res. 2007;40:663-70.

15. Abe F, Nagafuji S, Okawa M, Kinjo J, Akahane H, Ogura T, et al. Trypanocidal constituents in plants. 5. Evaluation of some Mexican plants for their trypanocidal activity and active constituents in the seeds of Persea americana. Biol pharm Bull. 2005;28(7):1314-7.

16. Truiti M, Ferreira I, Zamuner M, Nakamura C, Sarragiotto M, Souza M. Antiprotozoal and molluscicidal activities of five Brazilian plants. Braz J Med Biol Res. 2005;38:1873-8. 
17. Leite J, Oliveira A, Lombardi J, Filho J, Chiari E. Trypanocidal activity of triterpenes from Arrabidaea triplinervia and derivatives. Biol Pharm Bull. 2006;29(11):2307-9.

18. Sanchez A, Jimenez V, Sartor T, Tonn C, García E, Nieto M. A novel icetexane diterpene, 5-epiicetexone from Salvia gilliessi is active against trypanosoma cruzi. Acta Trop. 2006;98(2):11824.

19. Cunha W, Crevelin E, Arantes G, Crotti A, Andrade $M$, Furtado $M$, et al. A study of the trypanocidal activity of triterpene acids isolated from Miconia species. Phytother Res. 2006;20(6):474-8.

20. Jimenez V, Brengio S, Giordano 0, Tonn C, Sanchez M, Burgos M, et al. The trypanocidal effect of sesquiterpene lactones helenalin and mexicanin on cultured epimastigotes. J Parasitol. 2005;91(1):170-4.

21. González P, Marín C, Rodríguez-González I, Illana A, Mateo E, Longoni S, et al. Diterpenoid alkaloid derivatives as potencial chemotherapeutic agents in American Trypanosomiasis. Pharmacology. 2006;76:123-8.

22. Ferreira $M$, Nakayama $H$, De Arias $A$, Schinini A, De Bilbao A, Serna N, et al. Effects of canthin-6-one alkaloids from Zanthoxylum chiloperone on Trypanosoma cruzi-infected mice. J Ethnopharmacol. 2007;109(2):258-63.

23. Fournet A, Inchausti A, Yaluff $G$, Rojas de Arias A, Guinaudeau H, Bruneton J. Trypanocidal bisbenzylisoquinoline alkaloids are inhibitors of trypanothione reductase. J Enzyme Inhib. 1998;13(1):1-9.

24. Tasdemir D, Kaiser M, Brun R, Yardley V, Schmidt T, Tosun F, Rüedi P. Antitrypanosomal and antileishmanial activities of flavonoids and their analogues: in vitro, in vivo, structure-activity relationship, and quantitative structure-activity relationship studies. Antimicrobial Agents and Chemotherapy. 2006;50(4):1352-64.
25. Weniger B, Vonthron-Sénécheau C, Kaiser M, Brun R, Anton R. Comparative antiplasmodial, antileishmanicidal and antitrypanosomal activities of several biflavonoids. Phytomedicine. 2006;13:176-80.

26. Ribeiro A, Piló-Veloso D, Romaha A, Zani C. Trypanocidal flavonoids from Trixis vauthieri. J Nat Prod. 1997;60(8):836-41.

27. Da Silva F, Albuquerque S, Silva M, Eberlin M, Tomazela D, Bastos J. Tetrahydrofuran lignans from Nectandra megapotamica with trypanocidal activity. J Nat Prod. 2004;67(1):42-5.

28. Mishina Y, KrishnaS, Haynes R, Meade J. Artemisinins inhibit Trypanosoma cruzi and Trypanosoma brucei rhodesiense in vitro growth. Antimicrob Agents Chemother. 2007;51(5):1852-4.

29. Santoro G, Das GraÇas M, Guimaraes L, Salgado A, Mena R, Soares M. Effect of orégano (Origanum vulgare L.) and Thyme (Thymus vulgaris L.) essential oils on Trypanosoma cruzi (Protozoa: Kinetoplastida) growth and ultraestructure. Parasitol Res. 2007;100(4):783-90.

30. Santoro G, Cardoso M, Guimaraes L, Mendonça L. Soares M. Trypanosoma cruzi: activity of essential oils from Achillea millefolium L., Syzygium aromaticum $\mathrm{L}$. and 0 cimun basilicum $\mathrm{L}$. on epimastigotes and trypomastigotes. Exp Parasitol. 2007;116(3):283-90.

31. Tchoumbougnang F, Zollo P, Dagne E, Mekonnen $Y$. In vivo antimalarial activity of essential oils from Cymbopogon citratus and Ocimum gratissimum on mice infected with Plasmodium berghei. Planta Med. 2005;71(1):20-3.

32. Fournet A, Rojas de Arias A, Ferreira M, Nakayama $\mathrm{H}$, Torres de Ortiz S, Schinini A, et al. Efficacy of the bisbenzylisoquinoline alkaloids in acute and chronic Trypanosoma cruzi murine model. Int J Antimicrob Agents. 2000;13(3):189-95.

33. Menna R, Henriques A, Pinto A, Morgado J, Soares M, De Castro S. Effect of a $\beta$-lapachone- derived naphthoimidazole on Trypanosoma cruzi: identification of target organelles. J Antimicrob Chemother. 2005;56:1034-41.

34. Uchiyama N, Kabututu Z, Kubata B, Kiuchi F, Ito M, Nakajima-Shimada J, et al. Antichagasic activity of komaroviquinone is due to generation of reactive oxygen species catalyzed by Trypanosoma cruzi old yellow enzyme. Antimicrob Agents Chemother. 2005;49(12): 5123-6.

35. Oliveira S, Fonseca S, Romão P, Ferreira S, Cunha F. Nitric oxide mediates the microbicidal activity of eosinophils. Mem Inst Oswaldo Cruz. 1997;92 Suppl II:233-5.

36. Jeong $H$, Sang $H$, Joong D, Hee $Y$, Yeon D, Tae J. Inhibitory effect of citral on NO production by suppression of iNOS expression and NF- $\kappa \mathrm{B}$ activation in RAW264.7 cells. Arch Pharm Res. 2008;31(3):342-9.

37. Villalta F, Kierszenbaum F. Role of inflammatory cells in Chagas' disease. II. Interactions of mouse macrophages and human monocytes with intracellular forms of Trypanosoma cruzi: uptake and mechanism of destruction. J Immunol. 1984;133(6):3338-43.

Manuscrito recibido el 2 de setiembre de 2010 y aceptado para publicación el 30 de setiembre de 2010.

Correspondencia:

Juan Rojas Armas

Instituto de Investigaciones Clínicas, Hospital Nacional Dos de Mayo

Av. Grau cuadra 13, Parque Historia de la Medicina, Lima 1, Perú.

Correo electrónico: jprojasarmas@yahoo.com 